Nonlinear Processes in Geophysics (2002) 9: 69-74

Nonlinear Processes

in Geophysics

CE European Geophysical Society 2002

\title{
On resonant interactions of ions with plasma waves in a reduced quasi-linear theory
}

\author{
E. Marsch \\ Max-Planck-Institut für Aeronomie, Max-Planck-Str. 2, D-37191 Katlenburg-Lindau, Germany
}

Received: 12 June 2001 - Accepted: 30 July 2001

\begin{abstract}
Based on quasi-linear theory (involving pitch angle scattering), the resonant interactions between ions and waves in an anisotropic multi-component plasma are discussed. In particular, electromagnetic Alfvén and ioncyclotron waves propagating along or obliquely to the magnetic field are considered. A set of reduced (with respect to the perpendicular velocity component) quasi-linear diffusion equations is derived, involving reduced 1-D velocity distribution functions (VDFs), as they occur in wave dispersion relations. A 2-D model VDF can be constructed when using the Gaussian approximation. Wave-particle heating and acceleration rates are calculated.
\end{abstract}

\section{Introduction}

The purpose of this paper is to establish semi-kinetic, reduced diffusion equations for appropriately defined velocity distribution functions (VDFs) and to develop a set of comparatively simple closure relations, in order to describe the resonant interactions between ions and Alfvén waves, or oblique and dispersive ion-cyclotron and magnetosonic waves, as well as the interactions in Landau resonance of ions with the slow-mode and ion-acoustic waves in the solar wind and Sun's corona. This paper is an extension of the work by Marsch (1998) and Marsch and Tu (2001a) and is motivated by the clear evidence found by Marsch and Tu (2001b) for plateau formation in solar wind proton VDFs caused by diffusion in the cyclotron wave field. Furthermore, the spectroscopic determinations of the widths of extreme ultraviolet emission lines, as obtained from measurements made on the Solar and Heliospheric Observatory, indicate that heavy ions, coming in various ionization stages in the corona, are very hot (see, e.g. Kohl et al., 1997; Wilhelm et al., 1998; Cranmer et al., 1999), particularly in the polar coronal holes where electrons are rather cold. The ion kinetic temperatures seem to show some ordering with respect to the local gy-

Correspondence to: E. Marsch (marsch@linmpi.mpg.de) rofrequencies (Tu et al., 1998). All of these results hint to the importance of wave-particle processes.

It is well known that the protons in the high-speed solar wind reveal highly skewed and anisotropic VDFs, and that the heavy ions move faster and have higher temperatures than the protons. For reviews of these solar wind phenomena see, e.g. Marsch (1991), concerning the early in situ measurements made by Helios, and von Steiger et al. (1995), with respect to more recent observations of Ulysses. Often, the minor ion species can be considered as test particles, which probe the waves and turbulence in the wind and are heated and accelerated by wave-particle interactions. If the relative wave amplitudes are sufficiently small and the spectra broad in Fourier space, which is the case in the solar wind kinetic regime, then quasi-linear theory (QLT) (e.g. Stix, 1992) is adequate to describe the wave-particle couplings and the evolution of the particle VDFs, as well as the wave power spectral densities (PSDs).

\section{The quasi-linear diffusion equation}

Before we discuss the diffusion equation, we reiterate some of the basic equations and definitions needed subsequently. The QLT has been described originally by Kennel and Engelmann (1966) and then in many other articles. Here we refer mainly to the excellent textbooks by Melrose and McPhedran (1991) and Stix (1992). QLT is quadratically nonlinear in the coupling terms between the fluctuations of the VDFs and electromagnetic fields. But it is still linear (hence its name) in the sense that both kinds of fluctuations enter only linearly in the product terms of the perturbed second-order Vlasov equation. The wave properties, such as dispersion and polarization, are evaluated from linear theory with slowly timevarying VDFs and PSDs, implying weak wave growth or dissipation.

In QLT it is assumed that the electromagnetic wave fields can generally be Fourier-decomposed in plane waves with the frequency, $\omega=\omega_{M}(\mathbf{k})$, and growth rate, $\gamma_{M}(\mathbf{k})$, for a 
particular wave mode (index $M$ ) and a given wave vector $\mathbf{k}$, which is assumed here to be directed arbitrarily with respect to the constant background field, $\mathbf{B}_{0}$. The full dispersion equation for any linear plasma wave mode $M$ in a multicomponent plasma can be found, for instance, in Stix (1992). QLT assumes the validity of the random-phase approximation, which ensures that no constructive interference occurs between the different waves modes, and thus these modes can be simply superposed linearly. Therefore we can write the Fourier-transformed total electric field as a sum over the various modes as

$\tilde{\mathbf{E}}(\mathbf{k}, \omega)=2 \pi \sum_{M} \delta\left[\omega-\omega_{M}(\mathbf{k})\right] E_{M}(\mathbf{k}) \mathbf{e}_{M}(\mathbf{k})$.

The Fourier components of the electric field vector can be written in terms of the unimodular polarization vector, $\mathbf{e}_{M}(\mathbf{k})$. Mann et al. (1997) have recently studied in detail the polarization properties of waves in a multi-component plasma. The magnetic field, $\tilde{\mathbf{B}}_{M}(\mathbf{k})$, through the induction equation can be written as

$\tilde{\mathbf{B}}_{M}(\mathbf{k})=\frac{c}{\omega_{M}(\mathbf{k})} \mathbf{k} \times \tilde{\mathbf{E}}_{M}(\mathbf{k})$

The wave growth rate, $\gamma_{M}(\mathbf{k})$, or damping rate if it is negative, together with the real frequency, $\omega_{M}(\mathbf{k})$, may be combined to a complex frequency, $z_{M}(\mathbf{k})=\omega_{M}(\mathbf{k})+i \gamma_{M}(\mathbf{k})$. The spectral energy density of the magnetic field of mode $M$ is given by $\mathcal{B}_{M}(\mathbf{k})=\left|B_{M}(\mathbf{k})\right|^{2} /(8 \pi)$ and evolves according to

$\frac{\partial}{\partial t} \mathcal{B}_{M}(\mathbf{k})=2 \gamma_{M}(\mathbf{k}) \mathcal{B}_{M}(\mathbf{k})$

which follows from the Fourier decomposition

$\mathbf{B}_{M}(\mathbf{x}, t)=\int d^{3} k \tilde{\mathbf{B}}_{M}(\mathbf{k}) e^{i \mathbf{k} \cdot \mathbf{x}} e^{-i \int_{0}^{t} d t^{\prime} z_{M}\left(\mathbf{k}, t^{\prime}\right)}$,

where $\mathbf{x}$ is the spatial coordinate and $t$ is the time. One has $\tilde{\mathbf{B}}_{M}^{*}(\mathbf{k})=\tilde{\mathbf{B}}_{M}(-\mathbf{k})$, since the magnetic field in Eq. (4) must be real. Therefore, $\mathcal{B}_{M}(\mathbf{k})=\mathcal{B}_{M}(-\mathbf{k})$ by definition. The asterisk indicates the complex conjugate number. It is often convenient to use the Doppler-shifted frequency denoted by a prime, $\omega_{M}^{\prime}(\mathbf{k})=\omega_{M}(\mathbf{k})-k_{\|} U_{j}$, as measured in a frame of reference moving with the bulk speed component, $U_{j}$, of species $j$ along $\mathbf{B}_{0}$. The background electric field is taken to be zero, and the background plasma may be multicomponent but is assumed to bear zero current and be quasineutral.

In what follows the definitions are as usual: the speed of light is denoted by $c$, the ion charge by $e_{j}$, its density by $n_{j}$, the mass by $m_{j}$, and the plasma frequency of species $j$ is $\omega_{j}^{2}=\left(4 \pi e_{j}^{2} n_{j}\right) / m_{j}$. The ion gyrofrequency, carrying the sign of the charge, reads $\Omega_{j}=\left(e_{j} B_{0}\right) /\left(m_{j} c\right)$. Here the fractional mass density of species $j$ is defined as $\hat{\rho}_{j}=n_{j} m_{j} / \rho$, with $\rho=\sum_{\ell} n_{\ell} m_{\ell}$. We will make use of the relation $\hat{\rho}_{j} \Omega_{j}^{2}=\omega_{j}^{2} V_{A}^{2} / c^{2}$, where the Alfvén speed is based on the total mass density and defined as $V_{A}^{2}=B_{0}^{2} /(4 \pi \rho)$.
The quasi-linear diffusion equation describes the evolution of the velocity distribution function, $f_{j}\left(V_{\|}, V_{\perp}, t\right)$, of any particle species $j$ in an inertial frame of reference, in which the particles and waves are supposed to propagate. We will throughout the paper assume that the VDF is normalized to a density of unity. The general diffusion equation for any type of waves in a magnetized plasma and velocity distribution functions has originally been derived by Kennel and Engelmann (1966). It is calculated in a transparent way in the textbook of Stix (1992) and can be written as

$$
\begin{gathered}
\frac{\partial}{\partial t} f_{j}\left(V_{\|}, V_{\perp}, t\right)=\sum_{M} \sum_{s=-\infty}^{+\infty} \frac{1}{(2 \pi)^{3}} \int_{-\infty}^{+\infty} d^{3} k \hat{\mathcal{B}}_{M}(\mathbf{k}) \\
\quad \times \frac{1}{V_{\perp}} \frac{\partial}{\partial \alpha}\left(V_{\perp} v_{j}\left(\mathbf{k}, s ; V_{\|}, V_{\perp}\right) \frac{\partial}{\partial \alpha} f_{j}\left(V_{\|}, V_{\perp}, t\right)\right),
\end{gathered}
$$

where the pitch-angle gradient was introduced. It is given by the velocity derivative

$$
\frac{\partial}{\partial \alpha}=V_{\perp} \frac{\partial}{\partial V_{\|}}-\left(V_{\|}-\frac{\omega_{M}(\mathbf{k})}{k_{\|}}\right) \frac{\partial}{\partial V_{\perp}} .
$$

The magnetic field fluctuation spectrum is normalized to the background-field energy density. We find:

$\hat{\mathcal{B}}_{M}(\mathbf{k})=\left(\frac{B_{M}(\mathbf{k})}{B_{0}}\right)^{2}\left(\frac{k_{\|}}{k}\right)^{2} \frac{1}{1-\left|\hat{\mathbf{k}} \cdot \mathbf{e}_{M}(\mathbf{k})\right|^{2}}$.

The term in the denominator comes from the replacement of the electric field by the magnetic field power density. It turns out to be physically meaningful to introduce what we may call an ion-wave relaxation or collision rate. It is defined as

$$
\begin{aligned}
& v_{j}\left(\mathbf{k}, s ; V_{\|}, V_{\perp}\right)=\pi \Omega_{j}^{2} \delta\left(\omega_{M}(\mathbf{k})-s \Omega_{j}-k_{\|} V_{\|}\right) \\
& \quad \times\left|\frac{1}{2}\left(J_{s-1} e_{M}^{+}+J_{s+1} e_{M}^{-}\right)+\frac{V_{j}(\mathbf{k}, s)}{V_{\perp}} J_{s} e_{M z}\right|^{2} .
\end{aligned}
$$

Note that this quantity has indeed the dimension of an inverse time or rate. Here we also introduced the $s$-order resonance speed and made use of the Bessel functions (with index $s$ ):

$V_{j}(\mathbf{k}, s)=\frac{\omega_{M}(\mathbf{k})-s \Omega_{j}}{k_{\|}}, \quad J_{s}=J_{s}\left(\frac{k_{\perp} V_{\perp}}{\Omega_{j}}\right)$.

The circular components of the wave polarization vector are defined as

$e_{M}^{ \pm}(\mathbf{k})=e_{M x}(\mathbf{k}) \pm i e_{M y}(\mathbf{k})$.

The fundamental Eq. (5) is quoted here without derivation as the starting point of our paper. We have only slighty rewritten it in a form most appropriate for our subsequent purposes. Note that the famous quasi-linear plateau in the VDF implies a vanishing pitch-angle gradient, which means that $\partial / \partial \alpha=0$.

\section{Reduced velocity distributions and dispersive electro- magnetic waves}

For the general wave dispersion relation see any text book, such as Melrose and McPhedran (1991). To give a relevant 
and non-trivial example of a dispersion relation, we restrict ourselves in this section to parallel propagating electromagnetic waves. The full dispersion equation for parallel propagating left-handed $(M=-$ sign $)$ and right-handed $(M=+$ sign) circularly polarized electromagnetic waves in a multicomponent plasma reads, e.g. after Dum et al. (1980), as follows:

$$
\left(\frac{c k_{\|}}{z_{M}\left(k_{\|}\right)}\right)^{2}=1+\sum_{j}\left(\frac{\omega_{j}}{z_{M}\left(k_{\|}\right)}\right)^{2} \hat{\varepsilon}_{j}^{ \pm}\left(z_{M}^{\prime}\left(k_{\|}\right), k_{\|}\right)
$$

The dielectric constant involves a resonance integral over the pitch-angle gradient of the distribution function. Solving Eq. (11) for a given real $k_{\|}$, gives a complex frequency $z_{M}\left(k_{\|}\right)$. Usually there are at least as many modes or branches as there are species in the plasma considered (Mann et al., 1997). Expansion of Eq. (11) about the real axis allows one to derive the real and imaginary parts of the frequency (for small growth/damping rate) from the formulae

$$
\begin{aligned}
& \left(k_{\|} V_{A}\right)^{2}=\sum_{j} \hat{\rho}_{j} \Omega_{j}^{2} \operatorname{Re} \hat{\varepsilon}_{j}^{ \pm}\left(\omega_{M}^{\prime}\left(k_{\|}\right), k_{\|}\right), \\
& \gamma_{M}\left(k_{\|}\right)=\frac{-\sum_{j} \hat{\rho}_{j} \Omega_{j}^{2} \operatorname{Im} \hat{\varepsilon}_{j}^{ \pm}\left(\omega_{M}^{\prime}\left(k_{\|}\right), k_{\|}\right)}{\sum_{j} \hat{\rho}_{j} \Omega_{j}^{2} \frac{\partial}{\partial \omega_{M}\left(k_{\|}\right)}\left(\operatorname{Re} \hat{\varepsilon}_{j}^{ \pm}\left(\omega_{M}^{\prime}\left(k_{\|}\right), k_{\|}\right)\right)} .
\end{aligned}
$$

The Alfvén velocity has been used to normalize the phase speed, and terms of order $\left(V_{A} / c\right)^{2}$ have been neglected as being very small. The denominator of Eq. (13) is related to the so-called wave energy, associated with the sloshing motions of the particles in the wave field. The growth rate, $\gamma_{M}\left(k_{\|}\right)$, as obtained from Eq. (13), is to be used in Eq. (3) for the evolution of the wave spectrum.

The evaluation of the dielectric function in Eq. (11) does not require the full two-dimensional VDF but only the two reduced velocity distributions defined as follows:

$$
\begin{aligned}
& F_{j \|}\left(w_{\|}\right)=2 \pi \int_{0}^{\infty} d w_{\perp} w_{\perp} f_{j}\left(w_{\perp}, w_{\|}\right), \\
& F_{j \perp}\left(w_{\|}\right)=2 \pi \int_{0}^{\infty} d w_{\perp} w_{\perp} \frac{w_{\perp}^{2}}{2} f_{j}\left(w_{\perp}, w_{\|}\right) .
\end{aligned}
$$

In terms of these VDFs the dielectric function in Eq. (11) can after Dum et al. (1980) be written as

$$
\begin{aligned}
\hat{\varepsilon}_{j}^{ \pm} & =\int_{-\infty}^{\infty} \frac{d w_{\|}}{w_{j}^{ \pm}-w_{\|}}\left(\left(w_{\|}-\frac{z_{M}^{\prime}\left(k_{\|}\right)}{k_{\|}}\right) F_{j \|}\left(w_{\|}\right)\right. \\
& \left.+\frac{\partial}{\partial w_{\|}} F_{j \perp}\left(w_{\|}\right)\right) .
\end{aligned}
$$

Here we introduced the quantity $w_{j}^{ \pm}=\left(z_{M}^{\prime}\left(k_{\|}\right) \pm \Omega_{j}\right) / k_{\|}$, the real part of which is the resonance speed. Note that only the first quantity in Eq. (14) is a genuine particle VDF, whereas the second one in Eq. (15) corresponds to the distribution of the perpendicular thermal energy in dependence upon the parallel speed. Note further that by definition the following normalizations hold:

$\int_{-\infty}^{\infty} d w_{\|} F_{j \|}\left(w_{\|}\right)\left(\begin{array}{c}1 \\ w_{\|} \\ w_{\|}^{2}\end{array}\right)=\left(\begin{array}{c}1 \\ 0 \\ V_{j \|}^{2}\end{array}\right)$.

Due to the definition in Eq. (15), the zeroth moment of $F_{j \perp}$ yields the perpendicular thermal speed:

$\int_{-\infty}^{\infty} d w_{\|} F_{j \perp}\left(w_{\|}\right)=V_{j \perp}^{2}$.

Higher-order moments, such as heat fluxes, will not be considered here.

As a simple but most relevant example of the important "phase velocity", $V_{p h}(\mathbf{k})=\omega_{M}(\mathbf{k}) / k_{\|}$, appearing in Eq. (9), we consider here the phase speed of a parallel Alfvén wave. We start from the dispersion relation Eq. (12). An expansion of the dielectric constant for large resonant speeds, in the limit $\gamma_{M}\left(k_{\|}\right) \rightarrow 0$, in which $z_{M}^{\prime}\left(k_{\|}\right) \rightarrow \omega_{M}^{\prime}\left(k_{\|}\right)$, yields:

$V_{p h}=U \pm\left(V_{A}^{2}-\sum_{j} \hat{\rho}_{j}\left(V_{j \|}^{2}-V_{j \perp}^{2}+\Delta U_{j}^{2}\right)\right)^{1 / 2}$.

This is the phase velocity in the inertial frame of an Alfvén wave in a relatively drifting, anisotropic multi-ion plasma. The ion differential speed is $\Delta U_{j}=U_{j}-U$, with which each species moves along $\mathbf{B}_{0}$ relative to the center-of-mass frame, $U=\sum_{j} \hat{\rho}_{j} U_{j}$. The factor in the brackets of Eq. (19) is the generalized firehose correction. Alfvén waves have the unique property that $V_{p h}$ is constant and independent of the wave propagation angle.

\section{Coupled diffusion equations for the reduced distribu- tion functions}

We return to the general case of oblique wave propagation. In the diffusion Eq. (5) the particle speeds are given in the inertial frame. Let us now go into the proper frame of species $j$, in which the velocity is denoted by $\mathbf{w}$. It is obtained by replacing the velocity components as follows: $V_{\perp} \rightarrow w_{\perp}$, and $V_{\|} \rightarrow V_{\|}-U_{j}=w_{\|}$. The temporal rates of change of the reduced distribution functions are in this frame calculated by taking the corresponding moments of the diffusion Eq. (5) with the result:

$$
\begin{aligned}
\frac{\partial}{\partial t} F_{j \|} & =2 \pi \int_{0}^{\infty} d w_{\perp} w_{\perp} \frac{\partial}{\partial t} f_{j}\left(w_{\perp}, w_{\|}, t\right), \\
\frac{\partial}{\partial t} F_{j \perp} & =2 \pi \int_{0}^{\infty} d w_{\perp} w_{\perp} \frac{w_{\perp}^{2}}{2} \frac{\partial}{\partial t} f_{j}\left(w_{\perp}, w_{\|}, t\right) .
\end{aligned}
$$

By inserting Eq. (5) and partial integration with respect to $V_{\perp}$, the evolution equation for the parallel reduced VDF as defined in Eq. (14) is obtained

$$
\begin{gathered}
\frac{\partial}{\partial t} F_{j \|}\left(w_{\|}\right)=\frac{\partial}{\partial w_{\|}} D_{j}\left(w_{\|}\right) \frac{\partial}{\partial w_{\|}} F_{j \perp}\left(w_{\|}\right) \\
-\frac{\partial}{\partial w_{\|}}\left(A_{j}\left(w_{\|}\right) F_{j \|}\left(w_{\|}\right)\right) .
\end{gathered}
$$


It also depends on the reduced perpendicular VDF defined in Eq. (15), and thus the corresponding diffusion equation is also required, which reads:

$$
\begin{aligned}
& \frac{\partial}{\partial t} F_{j \perp}\left(w_{\|}\right)=\frac{\partial}{\partial w_{\|}} D_{j}\left(w_{\|}\right) \frac{\partial}{\partial w_{\|}} F_{j \perp}^{(2)}\left(w_{\|}\right) \\
& -2 \frac{\partial}{\partial w_{\|}}\left(A_{j}\left(w_{\|}\right) F_{j \perp}\left(w_{\|}\right)\right) \\
& \quad-A_{j}\left(w_{\|}\right) \frac{\partial}{\partial w_{\|}} F_{j \perp}\left(w_{\|}\right)+H_{j}\left(w_{\|}\right) F_{j \|}\left(w_{\|}\right) .
\end{aligned}
$$

In order to obtain these two diffusion equations, an important approximation had to be made in the relaxation rate $v_{j}$. It was taken at a typical value of $V_{\perp}$, for which we have chosen the mean thermal speed $V_{j \perp}$. Without this approximation, after partial integration of Eq. (5), one could have ignored the two reduced VFDs, but instead one would have had to deal with much more complex (weighted by Bessel functions) reduced VDFs. Such quantities are useless and have no obvious physical meaning. Since the matrix element occurring in Eq. (8) is positive definite, the original rate $v_{j}$ is expected to be a smoothly varying function of $V_{\perp}$, which we argue can be replaced without qualitative loss of essential physics, though quantitative loss of precision, by its typical value at the mean thermal speed. The resulting rate (including here another factor of two) is defined as

$$
\begin{aligned}
& \bar{v}_{j}\left(\mathbf{k}, s ; w_{\|}\right)=2 \pi \frac{\Omega_{j}^{2}}{\left|k_{\|}\right|} \delta\left(w_{\|}-V_{j}(\mathbf{k}, s)+U_{j}\right) \\
& \quad \times\left|\frac{1}{2}\left(\bar{J}_{s-1} e_{M}^{+}+\bar{J}_{s+1} e_{M}^{-}\right)+\frac{V_{j}(\mathbf{k}, s)}{V_{j \perp}} \bar{J}_{s} e_{M z}\right|^{2} .
\end{aligned}
$$

Note that this quantity has also the dimension of an inverse time or temporal rate. Here we used again the resonance speed Eq. (9) and introduced the slightly modified Bessel functions (with index $s$ ), depending only on $k_{\perp}$ in the form: $\bar{J}_{s}=J_{s}\left(k_{\perp} V_{j \perp} / \Omega_{j}\right)$.

Following Marsch (1998), we have defined the "transport" functions for the diffusion and acceleration, respectively, deceleration, and heating or cooling by the following wavevector integrals and sums over indices:

$$
\begin{array}{r}
\left(\begin{array}{c}
D_{j}\left(w_{\|}\right) \\
A_{j}\left(w_{\|}\right) \\
H_{j}\left(w_{\|}\right)
\end{array}\right)=\sum_{M} \frac{1}{(2 \pi)^{3}} \int_{-\infty}^{\infty} d^{3} k \hat{\mathcal{B}}_{M}(\mathbf{k}) \\
\times \sum_{s=-\infty}^{\infty} \bar{v}_{j}\left(\mathbf{k}, s ; w_{\|}\right)\left(\begin{array}{c}
1 \\
\frac{s \Omega_{j}}{k_{\|}} \\
\left(\frac{s \Omega_{j}}{k_{\|}}\right)^{2}
\end{array}\right) .
\end{array}
$$

These transport coefficients, which depend on $w_{\|}$only through the resonance condition in Eq. (24), describe the diffusive broadening of the VDFs and the particle's heating and acceleration, which ceases only when the particle co-moves with the wave in a frame defined by the velocity $V_{p h}(\mathbf{k})=\omega_{M}(\mathbf{k}) / k_{\|}$in the inertial frame. This result becomes obvious when one exploits the resonance condition in Eq. (24).
In Eq. (23) we were forced to introduce another higherorder reduced VDF given by

$F_{j \perp}^{(2)}\left(w_{\|}\right)=2 \pi \int_{0}^{\infty} d w_{\perp} w_{\perp} \frac{w_{\perp}^{4}}{4} f_{j}\left(w_{\perp}, w_{\|}\right)$,

for which in principle another evolution equation must be derived to obtain its time evolution. Apparently, we are facing the difficult problem of closure for the reduced VDFs. An infinite chain of evolution equations would result if no approximation was made. Therefore, the advantage of using 1-D VDFs instead of the original 2-D ones would be lost. To break this chain of higher-order moments we make the Gaussian approximation,

$F_{j \perp}^{(2)}\left(w_{\|}\right)=2 V_{j \perp}^{2} F_{j \perp}\left(w_{\|}\right)$,

which would be exact for a bi-Maxwellian. Of course, this does not imply that $F_{j \perp}$ is Gaussian itself. Empirical motivation for the factorization in Eq. (27) stems from the solar wind in situ observation, yielding that at any parallel speed the protons' perpendicular speeds are distributed as a Gaussian (Marsch and Goldstein, 1983), despite the fact that there may be proton beams (Marsch, 1991) drifting along the mean field. With the relation in Eq. (27) being inserted, the Eqs. (22 and (23) form a closed set of diffusion equations. Thus, Eq. (23) transforms into

$$
\begin{aligned}
\frac{\partial}{\partial t} F_{j \perp}\left(w_{\|}\right)=2 V_{j \perp}^{2} \frac{\partial}{\partial w_{\|}} D_{j}\left(w_{\|}\right) \frac{\partial}{\partial w_{\|}} F_{j \perp}\left(w_{\|}\right) \\
-3 A_{j}\left(w_{\|}\right) \frac{\partial}{\partial w_{\|}} F_{j \perp}\left(w_{\|}\right) \\
-2 F_{j \perp}\left(w_{\|}\right) \frac{\partial}{\partial w_{\|}} A_{j}\left(w_{\|}\right)+H_{j}\left(w_{\|}\right) F_{j \|}\left(w_{\|}\right) .
\end{aligned}
$$

The price to be payed for closure is that the evolution equation for $F_{j \perp}$ is now an integro-differential equation, since the squared perpendicular thermal speed is defined by the first parallel moment of $F_{j \perp}$. In a multi-component plasma, the dependence on $U_{j}$ is also essential, since it cannot be removed by going into the plasma (center-of-mass) frame. Stationary solutions of Eqs. (22) and (28) are obtained if the Gaussian condition,

$F_{j \perp}\left(w_{\|}\right)=V_{j \perp}^{2} F_{j \|}\left(w_{\|}\right)$,

is fulfilled, and if the only remaining reduced VDF obeys at the cyclotron resonance (for a given $k_{\|}$) the differential equation:

$V_{j \perp}^{2} \frac{\partial}{\partial w_{\|}} F_{j \|}=\frac{s \Omega_{j}}{k_{\|}} F_{j \|} \cdot$

Equations (29) and (30) express the conditions for a resonant plateau in terms of the reduced VDFs. In the case of nondispersive waves with a constant phase speed $V_{p h}$, Eq. (30) is valid everywhere in Fourier space and can therefore, by exploiting the resonance condition, be integrated to yield a reduced Maxwellian centered in the wave frame:

$F_{j \|}\left(w_{\|}\right)=\frac{1}{2 \pi V_{j \perp}^{2}} \exp \left(-\frac{\left(w_{\|}+U_{j}-V_{p h}\right)^{2}}{2 V_{j \perp}^{2}}\right)$. 
This result implies an $\alpha$-independent VDF, which then only depends on the particle energy in the wave frame, where its iso-density contours would be concentric circles.

The linked evolution Eqs. (22) and (28) are less complex than the original two-dimensional diffusion Eq. (5). This result is particularly advantageous if one seeks numerical solutions (see, e.g. the recent numerical model results obtained by Vocks and Marsch, 2001). Furthermore, according to these authors, one can construct a gyrotropic, approximate 2-D VDF by using the effective perpendicular thermal speed defined by:

$$
W_{j \perp}^{2}\left(w_{\|}\right)=\frac{F_{j \perp}\left(w_{\|}\right)}{F_{j \|}\left(w_{\|}\right)},
$$

which leads in consistency with the Gaussian approximation to a model VDF given by

$f_{j}\left(w_{\|}, w_{\perp}\right)=\frac{F_{j \|}\left(w_{\|}\right)}{2 \pi W_{j \perp}^{2}\left(w_{\|}\right)} \exp \left(-\frac{w_{\perp}^{2}}{2 W_{j \perp}^{2}\left(w_{\|}\right)}\right)$.

Illustrative examples of such re-constructed 2-D distributions for coronal funnels and holes can be found in the model results obtained by Vocks and Marsch (2001).

\section{Resonant heating and acceleration rates as reduced velocity moments}

Let us now take velocity moments of $\partial / \partial t F_{j \|, \perp}$ as given in Eqs. (22) and (28). Note that the reduced distribution functions vanish at infinity, which implies that $F_{j \|, \perp}( \pm \infty)=0$. The zeroth moment expresses conservation of normalization (or $n_{j}$ ). The first moment gives the bulk acceleration:

$$
\frac{\partial}{\partial t} U_{j}=<w_{\|} \frac{\partial F_{j \|}}{\partial t}>_{\|}
$$

The heating rates are defined by the second moments and given as:

$$
\begin{aligned}
\frac{\partial}{\partial t} V_{j \|}^{2} & =<w_{\|}^{2} \frac{\partial F_{j \|}}{\partial t}>_{\|}, \\
\frac{\partial}{\partial t} V_{j \perp}^{2} & =<\frac{\partial F_{j \perp}}{\partial t}>_{\|} .
\end{aligned}
$$

With the preparations of the previous sections, it is straightforward to evaluate the rates of change of the thermal speeds (or temperatures) and of the bulk speed of species $j$ by taking the first three parallel velocity moments of Eqs. (22) and (28), with the results:

$$
\begin{aligned}
& \frac{\partial}{\partial t} U_{j}=-<D_{j} \frac{\partial}{\partial w_{\|}} F_{j \perp}>_{\|}+<A_{j} F_{j \|}>_{\|} \\
& \frac{\partial}{\partial t} V_{j \|}^{2}=-2<w_{\|} D_{j} \frac{\partial}{\partial w_{\|}} F_{j \perp}>_{\|}+2<w_{\|} A_{j} F_{j \|}>_{\|} \\
& \frac{\partial}{\partial t} V_{j \perp}^{2}=-<A_{j} \frac{\partial}{\partial w_{\|}} F_{j \perp}>_{\|}+<H_{j} F_{j \|}>_{\|}
\end{aligned}
$$

Here the brackets with index $\|$ refer to an integration over $w_{\|}$only. These rates are, apart from the important approximations leading to Eq. (24), mathematically equivalent to the rates as given in Marsch and Tu (2001a), which were based on the original diffusion Eq. (5). In Eqs. (37) to (39) the character of the velocity moments is retained, and the waves appear only indirectly through the transport coefficients. Another version, stressing the waves, of the heating and acceleration rates is obtained by a direct integration over the wave spectrum. By evaluating the delta-function resonances explicitly, the equivalent form of the rates reads:

$$
\begin{aligned}
& \left(\begin{array}{l}
\frac{\partial}{\partial t} U_{j} \\
\frac{\partial}{\partial t} V_{j \|}^{2} \\
\frac{\partial}{\partial t} V_{j \perp}^{2}
\end{array}\right)=\sum_{M} \frac{1}{(2 \pi)^{3}} \int_{-\infty}^{+\infty} d^{3} k \hat{\mathcal{B}}_{M}(\mathbf{k})\left(\frac{\Omega_{j}}{k_{\|}}\right)^{2} \\
& \times \sum_{s=-\infty}^{+\infty} \overline{\mathcal{R}}_{j}(\mathbf{k}, s)\left(\begin{array}{c}
k_{\|} \\
2 k_{\|}\left(V_{j}(\mathbf{k}, s)-U_{j}\right) \\
s \Omega_{j}
\end{array}\right),
\end{aligned}
$$

where we used explicitly the wave spectrum $\hat{\mathcal{B}}_{M}(\mathbf{k})$, which is given by Eq. (7). Equation (40) expresses the rates in terms of an integral over the normalized PSD and sums over the mode number, $M$, and resonance-order number, $s$, and the reduced velocity distribution functions. The resonance function $\overline{\mathcal{R}}_{j}(\mathbf{k}, s)$ also depends on these numbers, and on the wave vector $\mathbf{k}$ and of course the reduced VDFs in the following form:

$$
\begin{aligned}
\overline{\mathcal{R}}_{j}(\mathbf{k}, s)=(2 \pi)^{2} \frac{k_{\|}}{\left|k_{\|}\right|} \\
\times\left[\left|\frac{1}{2}\left(\bar{J}_{s-1} e_{M}^{+}+\bar{J}_{s+1} e_{M}^{-}\right)+\frac{V_{j}(\mathbf{k}, s)}{V_{j \perp}} \bar{J}_{s} e_{M z}\right|^{2}\right. \\
\left.\quad \times\left(\frac{s \Omega_{j}}{k_{\|}} F_{j \|}-\frac{\partial}{\partial w_{\|}} F_{j \perp}\right)\right]_{w_{\|}=V_{j}(\mathbf{k}, s)-U_{j}} .
\end{aligned}
$$

The rates in Eq. (40) can be evaluated once $F_{j \perp, \|}\left(w_{\|}\right)$and the gyrotropic PSD, $\hat{\mathcal{B}}_{M}\left(k_{\perp}, k_{\|}\right)$, are known explicitly. Note that the quantity $\overline{\mathcal{R}}_{j}(\mathbf{k}, s)$ plays the role of a wave opacity, if one wants to use a technical term from radiative transfer theory. Other than in atomic optics, though, the opacity is here highly nonlinear, since it depends self-consistently on the reduced particle VDFs. At a given $k_{\|}$, the wave absorption vanishes on the plateau as defined by Eq. (30) for an isotropic VDF.

\section{Summary and conclusions}

We have first described in this article the general quasilinear interactions between ions and plasma waves propagating along or obliquely to the mean magnetic field. After that, a closure scheme for the semi-kinetic diffusion equations of the reduced VDFs has been established, by making the $V_{\perp}$-averaging assumption in the rate Eq. (24) and the Gaussian approximation leading to Eq. (27). Then the rates for the heating and acceleration of the ions, in cyclotron or Landau resonance with the waves, have been derived. The 
time evolution of the reduced VDFs is governed by the diffusion Eqs. (22) and (28), which require the knowledge of the wave transport coefficients in Eq. (25). They reflect through $V_{p h}(\mathbf{k})$ the actual wave dispersion properties, which often depend merely on the particles' temperatures, densities, and drift speeds, determining the characteristic frequencies and length scales.

If the complete physics of weak turbulence theory within the framework of QLT is to be retained, one needs to calcuate the dispersion and polarization properties of the waves selfconsistently, in order to calculate the required wave growth rate, $\gamma_{M}(\mathbf{k})$, and "phase speed" $V_{p h}(\mathbf{k})=\omega_{M}(\mathbf{k}) / k_{\|}$. Fortunately, convenient approximations for the polarization vectors exist, and many reasonable approximations of the dispersion relations (see, e.g. Stix, 1992; Melrose and McPhedran, 1991) can be derived, e.g. for a cold plasma, or a warm plasma with inclusion of substantial thermal corrections. Yet, more detailed dispersion relations, such as the one in Eq. (11), do require the reduced VDFs defined in Eqs. (14) and (15), or even the full distribution functions. For example, Dum et al. (1980) have numerically calculated the warm plasma dispersion relation for parallel waves based on measured solar wind particle VDFs.

The set of closure equations established here has already been demonstrated by Vocks (2001) and Vocks and Marsch (2001) to provide reasonable solutions and model VDFs for coronal holes. There are other obvious applications to the interplanetary solar wind, in which evidence for pitch-angle diffusion of protons has recently been found (Marsch and $\mathrm{Tu}, 2001$ ), the explanation of which requires a semi-kinetic or kinetic approach. It should be noted that our set of reduced equations can also be applied in a self-consistent scenario when the wave spectra and particle VDFs are time dependent. We think that the reduced semi-kinetic QLT is a promising step from a fluid-type towards a full kinetic description of wave-particle interactions in space plasmas.

\section{References}

Cranmer, S. R., Field, G. B., and Kohl, J. L.: Spectroscopic constraints on models of ion cyclotron resonance heating in the polar solar corona and high-speed solar wind, Astrophys. J., 518, 937, 1999.
Dum, C. T., Marsch, E., and Pilipp, W. G.: Determination of wave growth from measured distribution functions and transport theory, J. Plasma Phys., 23, 91, 1980.

Kennel, C. F. and Engelmann, F.: Velocity space diffusion from weak plasma turbulence in a magnetic field, Phys. Fluids, 9, 2377, 1966.

Kohl, J. L., Noci, G., Antonucci, E., et al.: First results from the SOHO UltraViolet Coronagraph Spectrometer, Solar Phys., 175, 613, 1997.

Mann, G., Hackenberg, P., and Marsch, E.: Linear mode analysis in multi-ion plasmas, J. Plasma Phys., 58, 205, 1997.

Marsch, E.: Kinetic physics of the solar wind, in: Physics of the Inner Heliosphere, (Eds) Schwenn, R. and Marsch, E., Springer Verlag, Heidelberg, Germany, Vol. II, 45, 1991

Marsch, E.: Closure of multifluid and kinetic equations for cyclotron-resonant interactions of solar wind ions with Alfvén waves, Nonl. Proc. Geophys., 5, 111, 1998.

Marsch, E. and Goldstein, H.: The effects of Coulomb collisions on solar wind ion velocity distributions, J. Geophys. Res., 88, 9933, 1983.

Marsch, E. and Tu, C. Y.: Heating and acceleration of coronal ions interacting with plasma waves through cyclotron and Landau resonance, J. Geophys. Res., 106, 227, 2001 a.

Marsch, E. and Tu, C. Y.: Evidence for pitch-angle diffusion of solar wind protons in resonance with cyclotron waves, J. Geophys. Res., 106, 8357, 2001b.

Melrose, D. B. and McPhedran, R. C.: Electromagnetic Processes in Dispersive Media, Cambridge Univiversity Press, New York, 1991.

Stix, T. H.: Waves in Plasmas, American Institute of Physics, New York, 1992.

Tu, C. Y., Marsch, E., Wilhelm, K., and Curdt, W.: Ion temperatures in a solar polar coronal hole observed by SUMER on SOHO, Astrophys. J., 500, 475, 1998

Vocks, C. and Marsch, E.: A semi-kinetic model of wave-ion interaction in the solar corona, Geophys. Res. Lett., 28, 1917, 2001.

Vocks, C.: Ein kinetisches Modell der Ionen in koronalen Löchern mit Welle-Teilchen-Wechselwirkung und Coulomb-Stößen, $\mathrm{PhD}$ Thesis, University of Göttingen, Göttinger Beiträge zur Physik, Band 8, Duerkohp \& Radicke, Wissenschaftliche Publikationen, Germany, 2001.

von Steiger, R., Geiss, J., Gloeckler, G., and Galvin, A. B.: Kinetic properties of heavy ions in the solar wind from SWICS/Ulysses, Space Science Rev., 72, 71, 1995.

Wilhelm, K., Marsch, E., Dwivedi, B. N., et al.: The solar corona above polar coronal holes as seen by SUMER on SOHO, Astrophys. J., 500, 1023, 1998. 